\title{
Nucleosynthesis in Jets from A Collapsar before The Formation of A Black Hole
}

\author{
Shin-ichiro Fujimoto* \\ Kumamoto National College of Technology, Kumamoto 861-1102, Japan \\ E-mail: fujimotodec.knct.ac.jp
}

Nobuya Nishimura, Masa-aki Hashimoto,

Department of Physics, School of Sciences, Kyushu University, Fukuoka 810-8560, Japan

Kei Kotake

Division of Theoretical Astronomy, National Astronomical Observatory Japan, 2-21-1, Osawa, Mitaka, Tokyo, 181-8588, Japan

\section{Shoichi Yamada}

Science \& Engineering, Waseda University, 3-4-1 Okubo, Shinjuku, Tokyo 169-8555, Japan

\begin{abstract}
We have investigated nucleosynthesis inside the jets from a $35 M_{\odot}$, low-metallicity collapsar before the formation of a black hole, based on a two-dimensional magneto-hydrodynamic (MHD) simulation of the collapsar, taking into account neutrino interactions both on the MHD simulation and nucleosynthesis. We find that jets are ejected via the magnetic pressure, and that $r$-process operates in the jets even if the neutrino absorption is taken into account in the nucleosynthesis calculation. This is due to efficient electron captures in the high-density $\left(\sim 10^{11} \mathrm{~g} \mathrm{~cm}^{-3}\right)$ region near the rotational axis, fast ejection ( $>0.2$ in units of speed of light), and relatively low neutrino luminosity $\left(<5 \times 10^{52} \mathrm{ergs} \mathrm{s}^{-1}\right)$. Chemical composition of the neutron-rich nuclei in jets from collapsars with different mass and rotation could be largely different from each other.
\end{abstract}

10th Symposium on Nuclei in the Cosmos

July 27 - August 1, 2008

Mackinac Island, Michigan, USA

* Speaker. 


\section{Introduction}

For a star more massive than $35-40 M_{\odot}$, the stellar core is considered to promptly collapse to a black hole during the collapse. When the star has sufficiently high angular momentum before the collapse, an accretion disk is formed around the black hole and jets are shown to be launched from the inner region of the disk through magnetic processes [1]. Gamma-ray bursts (GRBs) are expected to be driven by the jets. This scenario of GRBs is referred to as the collapsar model [2], which seems to be promising, because of the accumulating observations that imply an association between GRBs and the deaths of massive stars. Such the rotating massive star is called as a collapsar.

Although various astrophysical objects have been proposed as a $r$-process site, there is no compelling condidates [3]. It has been suggested that a collapsar could produce $r$-elements [4, 5]. We have shown that $r$-process successfully operates in the jets from collapsars, in particular jets with high energies [6, 7]. Although we investigated nucleosynthesis in the jets after the formation of a black hole in our previous study, nucleosynthesis in the jets before the black-hole formation could be different from that after the formation due to the presence of a proto neutron-star [8]. Moreover, recent elaborate simulations of the spherical collapse of a $40 M_{\odot}$ star has shown that the collapse of the stellar core to a black hole takes $0.6-1.3 \mathrm{~s}$ after the core bounce [9]. In the present study, we perform a two-dimensional simulation of a collapsar before the formation of the black hole. Then, we calculate the chemical composition of jets from the collapsar to investigate differences of the chemical composition of jets ejected before and after the black-hole formation.

\section{MHD Simulation of A Collapsar}

We have carried out Newtonian magneto-hydrodynamic (MHD) calculation of the collapse of a rotating, magnetized massive star. The numerical code for the MHD calculations employed in this paper is based on that used in the study on aspherical core collapse of a star [10]. The self-gravity of the star is taken into account. We use a realistic equation of state (EOS) based on the relativistic mean field theory [11] and an another EOS [12] for lower density regime $\left(\rho<10^{5} \mathrm{~g} / \mathrm{cm}^{3}\right)$, where no data is available in the EOS table with the Shen EOS. The use of the realistic EOSs is important for nucleosynthesis, because its enable us to evaluate temperatures of fluid preciously. Not as in the previous work [1, 6, 7], we follow the evolution of electron fraction, taking into account the change in the fraction due to electron and positron capture and neutrino and anti-neutrino absorption in terms of a leakage scheme [13].

Calculations are performed over the central region of a massive star with spherical coordinates, $(r, \theta, \phi)$. The computational domain is extended over $0 \mathrm{~km} \leq r \leq 5000 \mathrm{~km}$ and $0 \leq \theta \leq \pi / 2$ and covered with $300(r) \times 48(\theta)$ meshes. We assume that the fluid is axisymmetric and the mirror symmetry on the equatorial plane.

We set the initial profiles of the density, temperature and electron fraction to those of the 1.5 dimensional model of a $35 M_{\odot}$ massive star before the collapse, or 35OC model [14], which is a low-metallicity star $\left(0.1 Z_{\odot}\right)$ with a low mass-loss rate during its Wolf-Rayet phase. We note that evolution of radial distribution of angular velocity and magnetic fields is taken into account in the model [14]. We adopt an initial distribution of angular velocity as that of 35OC model. 
Initial magnetic field is assumed to be uniform with a vertical component of $10^{11} \mathrm{G}$ and a toroidal component of $8 \times 10^{11} \mathrm{G}$. We note that the toroidal component is comparable to that in $35 \mathrm{OC}$ model, while the vertical one is about a factor of five larger. It should be noted that the magnetic pressure is much smaller than the other pressure.

We briefly summarize results of the MHD simulation of the collapsar. Central iron core collapses and then bounces at $380 \mathrm{~ms}$ after the start of the simulation. Just after the core bounce, neutrino burst occurs and then luminosities of all flavors of neutrinos decrease to $<6 \times 10^{52} \mathrm{ergs} \mathrm{s}^{-1}$, at the epoch of jet-launch ( $80 \mathrm{~ms}$ after the bounce). We note that the luminosities are less than those for spherical collapse because of lower mass accretion rates due to rotation. The jets are ejected via the magnetic pressure, which is amplified via field wrapping and magneto-rotational instability. We find the jets ejected along the rotational axis $\left(<5\right.$ degree) have high density $\left(>10^{11} \mathrm{~g} \mathrm{~cm}^{-3}\right)$ above the neutrino sphere.

\section{Nucleosynthesis in collapsar}

In order to calculate chemical composition of material inside the jets, we need Lagrangian evolution of physical quantities, such as density, temperature, and, velocity of the material. We adopt a tracer particle method, as in [6, 7], to calculate the Lagrangian evolution of the physical quantities from the Eulerian evolution obtained from the MHD simulation of the collapsar. Particles are initially placed from an iron core to an inner O-rich layer. The numbers of the particles in a layer are weighted to the mass in the layer. The total number of the particles is set to be 3000 . The numbers of the particles in a layer are weighted to the mass of the layer. We find 213 jet particles that are particles ejected through the jets.
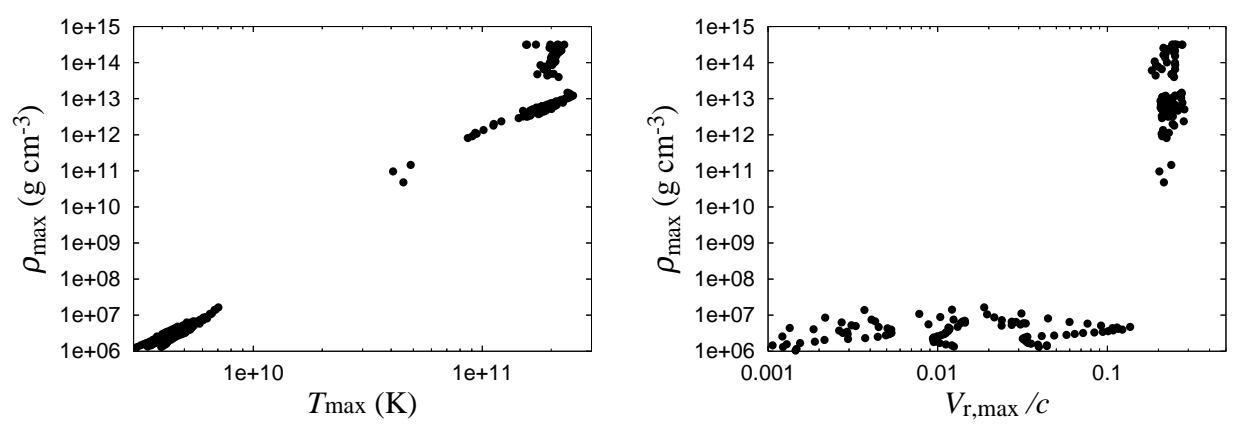

Figure 1: Maximum density vs maximum temperature (left panel) and maximum density vs maximum radial velocity (right panel) of all the jet particles.

Figure 1 shows maximum density as a function of maximum temperature (left panel) or maximum radial velocity (right panel) of all the jet particles. We find that a large fraction of jet particles fall inside the proto-neutron star, where the particles attain to very high densities $\left(>10^{11} \mathrm{~g} \mathrm{~cm}^{-3}\right)$ and temperatures $\left(>10^{11} \mathrm{~K}\right.$ ), and are in $\beta$-equilibrium. For densities $>10^{11} \mathrm{~g} \mathrm{~cm}^{-3}$, we set electron fraction of these jet particles to be the fraction taken from the MHD simulation, in which the evolution of electron fraction is taken into account with the leakage scheme. During the ejection through the jets, the density of the particles decreases, and $\beta$-equilibrium cannot maintain. In these relatively low-density region, we need to evaluate the evolution of electron fraction carefully. In the 
present study, we have estimated change in the electron fraction, taking into account the capture of electrons and positrons on nucleons and the absorption of neutrinos and anti-neutrinos via nucleons for densities $<10^{11} \mathrm{~g} \mathrm{~cm}^{-3}$. The rate of the absorption is calculated with neutrino and anti-neutrino number flux, which is globally evaluated from the distribution of density, temperature, and electron fraction taken from the MHD simulation, at the particle position.

We set chemical composition of a jet particles with temperature $>9 \times 10^{9} \mathrm{~K}$ to be that in nuclear statistical equilibrium (NSE), whose abundances are expressed with simple analytical expressions, specified by the density, temperature and electron fraction. In the relatively cool regime $<9 \times 10^{9} \mathrm{~K}$, NSE breaks and the chemical composition is calculated with a nuclear reaction network, which includes about 4000 nuclei from neutron and proton up to Fermium (see NETWORK $\mathrm{B}$ of Table 1 in [8]). The experimental masses and reaction rates are adopted if available or theoretical masses and rates are taken into account otherwise (see [6] for detailed nuclear data). Moreover, spontaneous and $\beta$-delayed fission and the asymmetric distribution of fission yields are taken into account in the network.

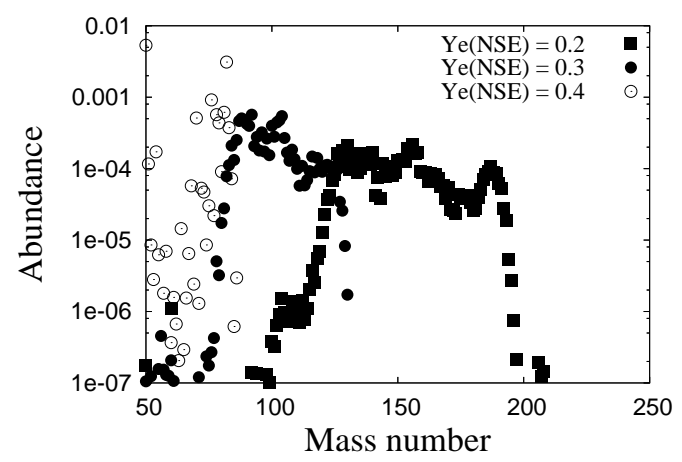

Figure 2: Abundances of three neutron-rich jet particles with $Y_{e}(\mathrm{NSE})=0.2$ (filled squares), 0.3 (filled circles), and 0.4 (open circles) after decays.

We find that there exist neutron-rich jet particles with $Y_{e}(\mathrm{NSE})<0.4$, where $Y_{e}(\mathrm{NSE})$ is the electron fraction of a jet particle at temperature of $9 \times 10^{9} \mathrm{~K}$. In particular, jet particles with $Y_{e}(\mathrm{NSE})<0.3$ are ejected along the rotational axis $(<5$ degree $)$. This is due to efficient electron captures in the high-density $\left(\sim 10^{11} \mathrm{~g} \mathrm{~cm}^{-3}\right)$ region near the rotational axis, fast ejection $(>0.2$ in units of speed of light; see right panel in Fig. 1), and relatively low neutrino luminosity $\left(<5 \times 10^{52} \mathrm{ergs} \mathrm{s}^{-1}\right)$.

Figure 2 shows abundances after decays of three neutron-rich jet particles with $Y_{e}(\mathrm{NSE})=0.2$ (filled squares), 0.3 (filled circles), and 0.4 (open circles). We find that $r$-process operates in jet particles with $Y_{e}(\mathrm{NSE}) \sim 0.2$ to produce $\mathrm{U}$ and Th. It should be noted that $r$-process operates in the collapsar jets even if the neutrino absorption is taken into account. Hence, neutrinos cannot kill the $r$-process. However, nuclei with mass number $<180$ and atomic number $<80$ are unlikely to be abundant, because of a small amount of the jet particles with $Y_{e}(\mathrm{NSE}) \sim 0.2$. We emphasize that jets from collapsars after the black-hole formation have abundant neutron-rich ejecta with $Y_{e}(\mathrm{NSE})<0.2$ [6, 7], in which $\mathrm{U}$ and Th are synthesized abundantly. Abundance profile of the jets are similar to that of solar $r$-elements. 


\section{Summary}

We have investigated nucleosynthesis inside the jets from a $35 M_{\odot}$ collapsar before the formation of a black hole, based on two-dimensional MHD simulations of the collapsar, taking into account neutrino interactions both on the MHD simulation and nucleosynthesis calculation reasonably. We set initial condition of the collapsar as those of 35OC model [14], which is a lowmetallicity star $\left(0.1 Z_{\odot}\right)$ with a low mass-loss rate during its Wolf-Rayet phase, and in which the evolution of radial distribution of angular velocity and magnetic fields is taken into account.

We find that jets are ejected via the magnetic pressure, and that the jets ejected along the rotational axis $(<5$ degree $)$ have high density $\left(>10^{11} \mathrm{~g} \mathrm{~cm}^{-3}\right)$ above the neutrino sphere. We also find that $r$-process operates in jet particles with $Y_{e}(\mathrm{NSE}) \sim 0.2$ to produce $\mathrm{U}$ and Th, even if we take into account the neutrino absorption in the nucleosynthesis calculation. This is due to efficient electron captures in the high-density $\left(\sim 10^{11} \mathrm{~g} \mathrm{~cm}^{-3}\right)$ region near the rotational axis, fast ejection ( $>0.2$ in units of speed of light), and relatively low neutrino luminosity $\left(<5 \times 10^{52} \mathrm{ergs} \mathrm{s}^{-1}\right)$ caused by rotation of the progenitor (Sec. 2). Nuclei with mass number $<180$ and atomic number $<80$ are unlikely to be abundant.

Abundances of neutron-rich nuclei of collapsar jets are significantly affected by properties of the jets, such as density distribution and ejection speed, as well as properties of proto-neutron star, such as neutrino luminosities and distribution of the electron fraction. Therefore, chemical composition of the neutron-rich nuclei in the jets from collapsars with different mass and rotation could be largely different from each other.

\section{References}

[1] Fujimoto, S., Kotake, K., Yamada, S., Hashimoto, M., \& Sato, K. 2006, ApJ , 644, 1040

[2] Woosley, S. E. 1993, ApJ , 405, 273

[3] Arnould, M., Goriely, S., \& Takahashi, K. 2007, Phys. Rep. 450, 97

[4] Pruet, J., Thompson, T. A., \& Hoffman, R. D. 2004, ApJ , 606, 1006

[5] Fujimoto, S., Hashimoto, M., Arai, K., \& Matsuba, R. 2004, ApJ , 614, 817

[6] Fujimoto, S., Hashimoto, M., Kotake, K., \& Yamada, S. 2007, ApJ , 656, 382

[7] Fujimoto, S., Nishimura, N., \& Hashimoto, M. 2008, ApJ , 680, 1350

[8] Nishimura, S., Kotake, K., Hashimoto, M., Yamada, S., Nishimura, N., Fujimoto, S., \& Sato, K. 2006, ApJ , 642, 410

[9] Sumiyoshi, K., Yamada, S., \& Suzuki, H. 2007, ApJ , 667, 382

[10] Kotake, K., Sawai, H., Yamada, S., \& Sato, K. 2004, ApJ , 608, 391

[11] Shen, H., Toki, H., Oyamatsu, K., \& Sumiyoshi, K. 1998, Nucl. Phys. A., 637, 435

[12] Blinnikov, S. I., Dunina-Barkovskaya, N. V., \& Nadyozhin, D. K. 1996, ApJS , 106, 171

[13] Ruffert, M., Janka, H.-T., \& Schaefer, G. 1996, A\&A, 311, 532

[14] Woosley, S. E., \& Heger, A. 2006, ApJ , 637, 914 